doi:10.13108/2020-12-1-13

\title{
SOLVABILITY AND SMOOTHNESS OF SOLUTION TO VARIATIONAL DIRICHLET PROBLEM IN ENTIRE SPACE ASSOCIATED WITH A NON-COERCIVE FORM
}

\author{
S.A. ISKHOKOV, B.A. RAKHMONOV
}

\begin{abstract}
In the work we study the solvability of the variational Dirichlet problem for one class of higher order degenerate elliptic operators in an entire $n$-dimensional Euclidean space. The coefficients of the operator have a power-law degeneracy at the infinity. The formulation of the problem is related with integro-differential sesquilinear form, which may not satisfy the coercivity condition. Earlier, the variational Dirichlet problem for degenerate elliptic operators associated with noncoercive forms was studied mostly for a bounded domain by means of a method based on a finite partition of unity of the domain. In contrast to this, we employ a special infinite partition of unity of the entire Euclidean space of finite multiplicity.

The method used is based on techniques from the theory of spaces of differentiable functions of many real variables with a power weight. The boundary conditions in the problem are homogeneous in the sense that a solution to the problem is sought in a functional space in which the set of infinitely differentiable compactly supported functions is dense.

The differential operator depends on the complex parameter $\lambda$, and the existence and uniqueness of a solution of the variational Dirichlet problem is proved in the case as $\lambda$ belongs to a certain angular sector with a vertex at zero that contains the negative part of the real axis. Under additional conditions on the smoothness of the coefficients and the right-hand side of the equation, the differential properties of the solution are studied.
\end{abstract}

Keywords: variational Dirichlet problem, elliptic operator, power degeneration, noncoercive form, smoothness of a solution.

Mathematics Subject Classification: 35J35, 35D30, 35J40, 35J70, 46E35

\section{INTRODUCTION}

The solvability of a variational Dirichlet problem for various classes of degenere elliptic operators is well studied in the case, when the sesquilinear forms associated with the considered operators satisfy the coercitivity condition, see [1]-[8] and the references therein. The case of elliptic operators associated with non-coercive sesquilinear forms is technically complicated and is studied rather poor. This case was considered first by K.Kh. Boimatov in work [9] and later in works [10]-[18]. Except for works [13], [18], in the other cited works there were studied the operators defined in a bounded domain. while the operators considered in works [13], [18], were defined in unbounded domains very close to bounded ones; these were a limiting-cylindrical domain with a zero diameter at infinity and some of its generalizations.

In contrast to the above mentioned works, here we first consider degenerate elliptic operators defined on an entire $n$-dimensional Euclidean space $\mathbb{R}^{n}$ and associated non-coercive sesquilinear forms. Similar exterior Dirichlet problem for degenerate elliptic operators in the case of the associated coercive forms were studied earlier in works [2], [3]. The method we apply is based

S.A. Iskhokov, B.A. Rakhmonov, Solvability and smoothness of solution to variational DiRichlet PRoblem in ENTIRE SPACE ASSOCIATED WiTh A NON-COERCIVE FORM.

(C)Iskhokov S.A., Rakhmonov B.A. 2020.

Submitted September 2, 2019. 
on employing the theory of weighted functional spaces. This is why in the second section we provide necessary definitions of functional spaces with weights and formulate their main properties. In the third section we prove the unique solvability of the homogeneous variational Dirichlet problem. Here the homogeneous boundary condition are treated in the sense that the solution to the considered problem is sought in a functional space, in which the set of compactly supported infinitely differentiable functions is dense. In the fourth section we formulate the result on smoothness of a solution of the considered problem.

The technique developed in our work allows us to study similar issues for other types of unbounded domains like the exterior of an unbounded domain, a half-space, an infinite cylindrical domain, etc.

\section{FunCtional SpaCES}

Let $\mathbb{R}^{n}$ be an $n$-dimensional Euclidean space of points $x=\left(x_{1}, x_{2}, \ldots, x_{n}\right), k=$ $\left(k_{1}, k_{2}, \cdots, k_{n}\right)$ be a multi-index and $|k|=k_{1}+k_{2}+\cdots+k_{n}$ be the length of $k$. We denote by $u^{(k)}(x)$ a generalized derivative in the Sobolev sense of the function $u(x)$. Let $d(x)=\left(1+|x|^{2}\right)^{-\frac{1}{2}}$, $r$ be a natural number, $\alpha, \delta, p$ be real numbers and $1 \leqslant p<\infty$. By the symbol $W_{p, \alpha, \delta}^{r}\left(\mathbb{R}^{n}\right)$ we denote the space of the functions $u(x)$ defined on the entire space $\mathbb{R}^{n}$ and having all generalized derivative in the Sobolev sense of order $r$ with a finite norm

$$
\left\|u ; W_{p, \alpha, \delta}^{r}\left(\mathbb{R}^{n}\right)\right\|=\left\{\left\|u ; L_{p, \alpha}^{r}\left(\mathbb{R}^{n}\right)\right\|^{p}+\left\|u ; L_{p, \delta}\left(\mathbb{R}^{n}\right)\right\|^{p}\right\}^{\frac{1}{p}}
$$

where

$$
\begin{aligned}
& \left\|u ; L_{p, \alpha}^{r}\left(\mathbb{R}^{n}\right)\right\|=\left\{\sum_{|k|=r} \int d^{p \alpha}(x)\left|u^{(k)}(x)\right|^{p} d x\right\}^{\frac{1}{p}}, \\
& \left\|u ; L_{p, \delta}\left(\mathbb{R}^{n}\right)\right\|=\left\{\int d^{p \delta}(x)|u(x)|^{p} d x\right\}^{\frac{1}{p}} .
\end{aligned}
$$

Hereinafter in the integral over the entire space $\mathbb{R}^{n}$ we omit the symbol $\mathbb{R}^{n}$. Together with the space $W_{p, \alpha, \delta}^{r}\left(\mathbb{R}^{n}\right)$ we define also spaces $W_{p, \alpha}^{r}\left(\mathbb{R}^{n}\right), V_{p, \alpha}^{r}\left(\mathbb{R}^{n}\right)$ respectively with the norms

$$
\begin{aligned}
& \left\|u ; W_{p, \alpha}^{r}\left(\mathbb{R}^{n}\right)\right\|=\left\{\left\|u ; L_{p, \alpha}^{r}\left(\mathbb{R}^{n}\right)\right\|^{p}+\int_{K_{R}}|u(x)|^{p} d x\right\}^{\frac{1}{p}}, \\
& \left\|u ; V_{p, \alpha}^{r}\left(\mathbb{R}^{n}\right)\right\|=\left\{\sum_{|k| \leqslant r} \int d^{p(\alpha+r-|k|)}(x)\left|u^{(k)}(x)\right|^{p} d x\right\}^{\frac{1}{p}} .
\end{aligned}
$$

Here $K_{R}$ is a ball of radius $R>0$ centered at the origin.

By $V_{q,-\alpha}^{-r}\left(\mathbb{R}^{n}\right)$, where $q=p /(p-1)$, we denote the space of antilinear continuous functionals $F$ over the space $V_{p, \alpha}^{r}\left(\mathbb{R}^{n}\right)$ equipped with the norm of dual space.

If $B$ is some of the spaces $W_{p, \alpha, \delta}^{r}\left(\mathbb{R}^{n}\right), W_{p, \alpha}^{r}\left(\mathbb{R}^{n}\right)$, the symbol $\stackrel{\circ}{B}$ stands for the closure of the set $C_{0}^{\infty}\left(\mathbb{R}^{n}\right)$ in the norm of this space.

Let us formulate the main properties of the space $V_{p, \alpha}^{r}\left(\mathbb{R}^{n}\right)$ implied by the corresponding results of works [4], [19], [20].

Theorem 2.1. For all $r \in \mathbb{N}, \alpha \in(-\infty,+\infty), p \in(1,+\infty)$ the following statements hold:

1) the set $C_{0}^{\infty}\left(\mathbb{R}^{n}\right)$ is dense in the space $V_{p, \alpha}^{r}\left(\mathbb{R}^{n}\right)$; 
2) norm (2.2) in the space $V_{p, \alpha}^{r}\left(\mathbb{R}^{n}\right)$ is equivalent to the following quantity:

$$
\left\|u ; V_{p ; \alpha}^{r}\left(\mathbb{R}^{n}\right)\right\|_{*}=\left\{\sum_{|k|=r} \int d^{p \alpha}(x)\left|u^{(k)}(x)\right|^{p} d x+\int d^{p(\alpha+r)}(x)|u(x)|^{p} d x\right\}^{\frac{1}{p}} ;
$$

3) for each natural number $m$ the embeddings hold:

$$
V_{p ; \alpha-m}^{r+m}\left(\mathbb{R}^{n}\right) \rightarrow V_{p ; \alpha}^{r}\left(\mathbb{R}^{n}\right), \quad V_{q,-\alpha-m}^{-r+m}\left(\mathbb{R}^{n}\right) \rightarrow V_{q,-\alpha}^{-r}\left(\mathbb{R}^{n}\right) .
$$

4) Let $n / p-\alpha \notin\{1,2, \ldots, r\}$. Then the identity holds:

$$
\stackrel{\circ}{W}_{p, \alpha}^{r}\left(\mathbb{R}^{n}\right)=V_{p, \alpha}^{r}\left(\mathbb{R}^{n}\right) .
$$

The space $W_{p, \alpha}^{r}\left(\mathbb{R}^{n}\right)$ was introduced by L.D. Kudryavtsev and it was studied in details in works [21], [22], see also [3].

In the next section, while estimating some auxiliary forms, we shall make use of Lemma 2.2 in [6]. Below we formulate this lemma in a convenient for us form for the spaces of differentiable functions of many variables in the entire space $\mathbb{R}^{n}$ with a power weight.

Lemma 2.1. Let $m \in[0, r)$ be an integer number, $p \geqslant 1,1 \leqslant q_{1} \leqslant q_{0}$, and a number $q_{0}$ satisfies the conditions

$$
\left\{\begin{array}{l}
\frac{1}{p}-\frac{r-m}{n}<\frac{1}{q_{0}}, \quad \text { as } n-(r-m) p>0 ; \\
q_{0} \text { is an arbitrary finite number, }
\end{array}\right.
$$

Then for each $\varepsilon>0$ and all $v \in V_{p, \alpha}^{r}\left(\mathbb{R}^{n}\right)$ the inequality

$$
\left\|v ; L_{q_{0}, \alpha-\frac{n}{p}+\frac{n}{q_{0}}+r-m}^{m}\left(\mathbb{R}^{n}\right)\right\| \leqslant \varepsilon\left\|v ; V_{p, \alpha}^{r}\left(\mathbb{R}^{n}\right)\right\|_{*}+c_{0} \varepsilon^{-\mu}\left\|v ; L_{q_{1}, \alpha-\frac{n}{p}+\frac{n}{q_{1}}+r}\left(\mathbb{R}^{n}\right)\right\|
$$

holds true, where

$$
\mu=\frac{q_{1}^{-1}-q_{0}^{-1}+m n^{-1}}{q_{0}^{-1}-p^{-1}+(r-m) n^{-1}} .
$$

\section{SOlvability OF HOMOgENEOUS VARIATIONAL DiRICHLET PROBLEM}

In this section we assume that $r$ is natural, $\alpha, \delta$ are real numbers satisfying the condition $\delta \leqslant \alpha+r$, and in order to simplify the writing, by $\stackrel{\circ}{H}_{+}$we denote the closure of the class $C_{0}^{\infty}\left(\mathbb{R}^{n}\right)$ in the norm of the space $H_{+}=W_{2, \alpha, \delta}^{r}\left(\mathbb{R}^{n}\right)$, while the symbol $\stackrel{\circ}{H}_{-}$stands for the completion of the space $H_{0}=L_{2, \delta}\left(\mathbb{R}^{n}\right)$ with respect to the norm

$$
\|f\|_{-}=\sup _{\substack{0 \neq u \in H_{+}\\}} \frac{\left|(f, u)_{\delta}\right|}{\|u\|_{+}} .
$$

Hereinafter $\|\cdot\|_{+}$is the norm in the space $H_{+}$and

$$
(f, u)_{\delta}=\int d^{2 \delta}(x) f(x) \overline{u(x)} d x,
$$

is the scalar product in $H_{0}$. The norm in the space $H_{0}$ is denoted by $\|\cdot\|_{\delta}$. The elements of the space $\stackrel{\circ}{H}_{-}$are identified with the corresponding antilinear continuous functionals over $\stackrel{\circ}{H}_{+}$. The action of the functional $F \in \stackrel{\circ}{H}_{-}$on a function $u \in \stackrel{\circ}{H}_{+}$is denoted $\langle F, u\rangle$. Thus, we obtain a triple of densely embedded spaces $\stackrel{\circ}{H}_{+} \rightarrow H_{0} \rightarrow \stackrel{\circ}{H}_{-}$. This triple is called a rigged Hilbert space, while $\stackrel{\circ}{H}_{+}$and $\stackrel{\circ}{H}_{-}$are called respectively positive and negative spaces [23, Ch. 1]).

We note that

$$
\left\|u ; V_{p ; \alpha}^{r}\left(\mathbb{R}^{n}\right)\right\|_{*} \leqslant\left\|u ; W_{p ; \alpha, \delta}^{r}\left(\mathbb{R}^{n}\right)\right\| \quad \text { as } \quad \delta \leqslant r+\alpha
$$


and by the equivalence of norms $(2.2)$ and $(2.3)$ as $\delta \leqslant \alpha+r$, the inequalities

$$
\int d^{2 \alpha+2 r-2|k|}(x)\left|u^{(k)}(x)\right|^{2} d x \leqslant M_{1}\|u\|_{+}
$$

hold for all $u \in \stackrel{\circ}{H}_{+}$and $|k| \leqslant r$; the number $M_{1}>0$ is independent of $u$.

In what follows by $M_{2}, M_{3}, \ldots$ we denote various positive inessential constants.

On functions $u, v \in C_{0}^{\infty}\left(\mathbb{R}^{n}\right)$ we consider a sesquilinear form

$$
B[u, v]=\sum_{|k|,|l| \leqslant r}\left(d^{-|k|} a_{k l} u^{(k)}, d^{-|l|} v^{(l)}\right)_{\alpha+r},
$$

whose coefficients $a_{k l}(x)$ are bounded complex-valued measurable functions. In what follows we study the solvability of the following variational Dirichlet problem associated with form 3.2 .

Problem $D_{\lambda}$. Given a functional $F \in \stackrel{\circ}{H}_{-}$, find a solution $u \in \stackrel{\circ}{H}_{+}$to the equation

$$
B_{\lambda}[u, v] \stackrel{\text { def }}{=} B[u, v]+\lambda(u, v)_{\delta}=<F, v>\quad \text { for all } \quad v \in C_{0}^{\infty}\left(\mathbb{R}^{n}\right) .
$$

We note that each solution of equation $(3.3)$ is called a generalized solution of the differential equation

$$
\sum_{|k|,|l| \leqslant r}(-1)^{|l|}\left(d^{2 r+2 \alpha-|k|-|l|}(x) a_{k l}(x) u^{(k)}(x)\right)^{(l)}+\lambda d^{2 \delta}(x) u(x)=F, \quad x \in \mathbb{R}^{n} .
$$

Together with form $(3.2)$, we introduce the function

$$
A(x, \zeta)=\sum_{|k|,|l| \leqslant r} a_{k l}(x) \zeta_{k} \overline{\zeta_{l}}
$$

defined for all $x \in \mathbb{R}^{n}$ and each set of complex numbers $\zeta=\left\{\zeta_{k}\right\}_{|k| \leqslant r}$.

Assume that for all $x \in \mathbb{R}^{n}$ and each set of complex numbers $\zeta=\left\{\zeta_{k}\right\}_{|k| \leqslant r}$ the conditions

$$
\begin{gathered}
|\arg A(x, \zeta)|<\varphi, \\
\sum_{|k|=r}\left|\zeta_{k}\right|^{2} \leqslant M_{2} \operatorname{Re}\{\gamma(x) A(x, \zeta)\},
\end{gathered}
$$

hold, where $\varphi$ is some number in the interval $(\pi / 2, \pi), \gamma(x)$ is everywhere continuous nonvanishing function with the following property: for each number $\nu>0$ there exists a number $R_{\nu}>0$ such that

$$
|\gamma(x)-\gamma(y)|<\nu
$$

for all $x, y \in \mathbb{R}^{n}$ such that $|x|>R_{\nu},|y|>R_{\nu}$. Hereinafter we assume that the function $\arg z$ ranges in $(-\pi, \pi]$.

We note that the study of the solvability of the variational Dirichlet problem associated with a non-coercive form made in works [9] 17] for a bounded domain was based on a finite partition of the unity in the considered domain. In contrast to that case, here we employ an infinite partition of the unity of the space $\mathbb{R}^{n}$ constructed in the following lemma.

Lemma 3.1. Let a function $\gamma(x), x \in \mathbb{R}^{n}$, satisfies condition (3.6) and let $\nu$ be a sufficiently small positive number. Then there exist non-negative functions $\varphi_{m} \in C_{0}^{\infty}\left(\mathbb{R}^{n}\right), \eta_{m} \in C_{0}^{\infty}\left(\mathbb{R}^{n}\right)$, $m=1,2, \ldots$, such that

a) the system of functions $\left\{\varphi_{m}^{2}(x)\right\}_{m=1}^{\infty}$ forms the partition of the unity in the space $\mathbb{R}^{n}$ with a finite multiplicity, that is,

$$
\sum_{m=1}^{\infty} \varphi_{m}^{2}(x) \equiv 1, x \in \mathbb{R}^{n},
$$


and if $\chi_{m}(x)$ is a characteristic function of the set $\operatorname{supp} \varphi_{m}$, then there exists a finite number $\Lambda_{n}$ depending on $n$ only such that

$$
1 \leqslant \sum_{m=1}^{\infty} \chi_{m}(x) \leqslant \Lambda_{n} \text { for all } x \in \mathbb{R}^{n}
$$

b) the function $\eta_{m}$ is identically equal to one in some neighbourhood of the set $\operatorname{supp} \varphi_{m}(x)$ and $0 \leqslant \eta_{m}(x) \leqslant 1$ for all $x \in \mathbb{R}^{n}$;

c) the derivatives of the functions $\varphi_{m}, \eta_{m}, m=1,2, \ldots$, obey the inequalities

$$
\left|\varphi_{m}^{(k)}(x)\right| \leqslant C_{1} d^{|k|}(x), \quad\left|\eta_{m}^{(k)}(x)\right| \leqslant C_{2} d^{|k|}(x),|k| \leqslant r,
$$

where positive numbers $C_{1}, C_{2}$ are independent of $m$ and $r$;

d) $|\gamma(x)-\gamma(y)|<\nu$ for all $x, y \in \operatorname{supp} \eta_{m}, m=1,2, \ldots$

Proof. Proceeding as in the proof of Lemma 7.1 of work [24], we construct the partition of the unity

$$
\sum_{m=1}^{\infty} \psi_{m}(x) \equiv 1, \quad x \in \mathbb{R}^{n}
$$

of multiplicity $\Lambda_{n} \leqslant(10)^{9 n}$, where non-negative functions $\psi_{1}, \psi_{2}, \ldots$, in $C_{0}^{\infty}\left(\mathbb{R}^{n}\right)$ satisfy the inequalities

$$
\begin{aligned}
& \left|\psi_{m}^{(k)}(x)\right| \leqslant C_{1}^{\prime} d^{|k|}(x), \quad x \in \mathbb{R}^{n}, m=1,2, \ldots \\
& |x-y| d(x) \leqslant 1 \quad x, y \in \operatorname{supp} \psi_{m}, m=1,2, \ldots
\end{aligned}
$$

We let

$$
\varphi_{m}(x)=\psi_{m}(x)\left(\sum_{j=1}^{\infty} \psi_{j}^{2}(x)\right)^{-\frac{1}{2}}, \quad \eta_{m}(x)=\sum^{\prime} \varphi_{j}(x)\left(\sum_{i=1}^{+\infty} \varphi_{i}(x)\right)^{-1}, \quad m=1,2, \ldots
$$

where $\sum^{\prime}$ denotes the summation over indices $j$ such that $\varphi_{m}(x) \varphi_{j}(x) \not \equiv 0$. These functions possess properties a) - d). Since a non-vanishing complex-valued function $\gamma(x)$ is everywhere continuous and for each number $\nu>0$, thanks to (3.6), there exists a number $R_{\nu}>0$ such that $|\gamma(x)-\gamma(y)|<\nu$ for all $x, y \in \mathbb{R}^{n}$ obeying $|x|>R_{\nu},|y|>R_{\nu}$, statement d) can fail for finitely many indices $m$. In this case, for the indices $m$, for which statement d) fails, we represent the functions $\varphi_{m}, \eta_{m}$ as finite sums of similar functions and we achieve in this way that statement d) holds for all $m=1,2, \ldots$

In what follows we shall employ Lemma 2.2 from [25]. We formulate this lemma in a convenient for us form.

Lemma 3.2. Let $\Lambda_{n}, \chi_{m}, m=1,2, \ldots$, be same as in Lemma 3.1. Let the operator $T$ reads as

$$
T=\sum_{m=1}^{+\infty} \chi_{m} T_{m} \chi_{m}
$$

where $T_{1}, T_{2}, \ldots$ is a sequence of continuous operators in $L_{p}\left(\mathbb{R}^{n}\right)$ such that

$$
\Lambda=\sup _{m=1,2, \ldots}\left\|T_{m}\right\|_{p}<+\infty
$$

where $p \in(1,+\infty)$ is some number. Then $T$ is a bounded operator and the inequality holds:

$$
\|T\|_{p} \leqslant \Lambda_{n}^{\frac{1}{p}} \Lambda \text {. }
$$

Here $\|T\|_{p}$ stands for the norm of a continuous operator $T: L_{p}\left(\mathbb{R}^{n}\right) \rightarrow L_{p}\left(\mathbb{R}^{n}\right)$. 
Now we are in position to formulate the main result of the present work.

Theorem 3.1. Let $\delta \leqslant \alpha+r$ and conditions (3.4)-(3.6) hold. Then there exists a sector $S \subset\{z \in C:|\arg z|<\pi-\varphi\} \cup\{0\}$ with the vertex at zero and a positive number $\sigma_{0}$ such that if $\lambda \in S$ and $|\lambda| \geqslant \sigma_{0}$, then for each given functional $F \in \stackrel{\circ}{H}_{-}$, Problem $D_{\lambda}$ is uniquely solvable and the estimate

$$
\|u\|_{+} \leqslant M_{3}\|F\|_{-}
$$

holds true, where a number $M_{3}>0$ is independent of $\lambda \in S$ and the functional $F$.

Proof. Let $\varphi_{m}, \eta_{m}, m=1,2, \ldots$ be the same functions as in Lemma 3.1. In each set $\operatorname{supp} \varphi_{m}, m=1,2, \ldots$, we fix a point $x_{m}$ and we consider the form

$$
B_{\lambda ; m}^{(0)}[u, v]=\sum_{|k|,|l| \leqslant r}\left(d^{-|k|} a_{k l m}^{(0)} u^{(k)}, d^{-|l|} v^{(l)}\right)_{\alpha+r}+\lambda(u, v)_{\delta},
$$

where

$$
a_{k l m}^{(0)}(x)=\left(1-\eta_{m}(x)\right) \gamma\left(x_{m}\right) a_{k l}\left(x_{m}\right)+\eta_{m}(x) \gamma(x) a_{k l}(x) .
$$

The boundedness of the coefficients $a_{k l}(x),|k|,|l| \leqslant r$, implies the boundedness of the coefficients $a_{k l m}^{(0)}$. This is why, applying Cauchy-Bunyakovsky inequality and 3.1 , we get

$$
\begin{aligned}
\left|B_{\lambda ; m}^{(0)}[u, v]\right| \leqslant & M_{4} \int \sum_{|k|,|l| \leqslant r} d^{2 \alpha+2 r-|k|-|l|}(x)\left|u^{(k)}(x)\right|\left|v^{(l)}(x)\right| d x \\
& +|\lambda| \int d^{2 \delta}\left|u(x)\left\|v(x) \mid d x \leqslant M_{1}\left(M_{4}+|\lambda|\right)\right\| u\left\|_{+} \cdot\right\| v \|_{+}\right.
\end{aligned}
$$

for all $u, v \in \stackrel{\circ}{H}_{+}$.

If follows from condition (3.5) that

$$
\operatorname{Re}\left\{\sum_{|k|,|l| \leqslant r} a_{k l m}^{(0)}(x) \zeta_{i} \bar{\zeta}_{j}\right\} \geqslant c \sum_{|k|=r}\left|\zeta_{k}\right|^{2}
$$

for all $m=1,2,3, \ldots, x \in \mathbb{R}^{n}, \zeta=\left\{\zeta_{k}\right\}_{|k| \leqslant r} \subset C$. Substituting $\zeta_{k}=d^{\alpha+r-|k|}(x) u^{(k)}(x)$ into this inequality and integrating over $\mathbb{R}^{n}$, we get:

$$
\operatorname{Re} B_{\lambda ; m}^{(0)}[u, u] \geqslant C_{0}\|u\|_{+}^{2}, \quad \operatorname{Re} \lambda \geqslant 1
$$

for all $m=1,2,3, \ldots, u \in \stackrel{\circ}{H}_{+}$.

Now we consider a sesquilinear form

$$
\mathcal{B}_{\lambda ; m}^{(0)}[u, v]=\sum_{|k|,|l| \leqslant r}\left(d^{-|k|} \widehat{a}_{k l m} u^{(k)}, d^{-|l|} v^{(l)}\right)_{\alpha+r}+\lambda(u, v)_{\delta},
$$

where

$$
\widehat{a}_{k l m}(x)=\left[\left(1-\eta_{m}(x)\right) a_{k l}\left(x_{m}\right)+\eta_{m}(x) a_{k l}(x)\right] \gamma\left(x_{m}\right) .
$$

Since

$$
a_{k l m}^{(0)}(x)-\widehat{a}_{k l m}(x)=\eta_{m}(x)\left(\gamma(x)-\gamma\left(x_{m}\right)\right) a_{k l}(x),
$$

and the coefficients $a_{k l}(x)$ are bounded, we proceed as in the proof of inequality 3.10 to obtain

$$
\left|B_{\lambda ; m}^{(0)}[u, v]-\mathcal{B}_{\lambda ; m}^{(0)}[u, v]\right| \leqslant M_{5} \Lambda\|u\|_{+} \cdot\|v\|_{+}
$$

for all $u, v \in \stackrel{\circ}{H}_{+}$. Here $\Lambda=\sup \left|\eta_{m}(x)\left(\gamma(x)-\gamma\left(x_{m}\right)\right)\right|$, where the supremum is taken over all $x \in R_{n}$ and all $m=1,2,3, \ldots$ 
Applying this inequality, by (3.11) we find

$$
\begin{aligned}
C_{0}\|u\|_{+}^{2} & \leqslant \operatorname{Re}\left(B_{\lambda ; m}^{(0)}[u, u]-\mathcal{B}_{\lambda ; m}^{(0)}[u, u]\right)+\operatorname{Re} \mathcal{B}_{\lambda ; m}^{(0)}[u, u] \\
& \leqslant \operatorname{Re} \mathcal{B}_{\lambda ; m}^{(0)}[u, u]+M_{5} \Lambda\|u\|_{+}^{2}, \quad u \in \stackrel{\circ}{H}_{+} .
\end{aligned}
$$

Since

$$
\left|\eta_{m}(x)\left(\gamma(x)-\gamma\left(x_{m}\right)\right)\right|<\nu, \quad x \in \operatorname{supp} \eta_{m}, \quad m=1,2, \ldots,
$$

and $\nu$ is a sufficiently small positive number, it follows from 3.12 that

$$
c_{0}\|u\|_{+}^{2} \leqslant \operatorname{Re} \mathcal{B}_{\lambda ; m}^{(0)}[u, u], \quad \operatorname{Re} \lambda \geqslant 1, u \in \stackrel{\circ}{H}_{+} .
$$

We introduce the following sesquilinear form

$$
\mathcal{B}_{\lambda ; m}[u, v]=\sum_{|k|,|l| \leqslant r}\left(d^{-|k|} a_{k l m} u^{(k)}, d^{-|l|} v^{(l)}\right)_{\alpha+r}+\lambda(u, v)_{\delta},
$$

where

$$
a_{k l m}(x)=\left(1-\eta_{m}(x)\right) a_{k l}\left(x_{m}\right)+\eta_{m}(x) a_{k l}(x) .
$$

We note that $\gamma\left(x_{m}\right) \mathcal{B}_{\lambda ; m}[u, v]=\mathcal{B}_{\lambda_{m} ; m}^{(0)}[u, v]$, where $\lambda_{m}=\lambda \gamma\left(x_{m}\right)$. This is why it follows from inequality 3.13 that as $\operatorname{Re} \gamma\left(x_{m}\right) \lambda \geqslant 1$,

$$
c_{0}\|u\|_{+}^{2} \leqslant \operatorname{Re}\left\{\gamma\left(x_{m}\right) \mathcal{B}_{\lambda ; m}[u, u]\right\}, \quad u \in \stackrel{\circ}{H}_{+} .
$$

By (3.4), inequality (3.5) holds also in the case, when we replace $\gamma(x)$ by $\exp (i \theta(x))$, where

$$
\theta(x)=\min \left\{\varphi-\frac{\pi}{2},|\arg \gamma(x)|\right\}(\operatorname{sign} \arg \gamma(x)) .
$$

By $S$ we denote a closed angular sector with the vertex at the origin on the complex plane such that $|\theta(x)+\arg z|<\pi / 2$ for all $x \in \mathbb{R}^{n}, z \in S$. We note that

$$
S \subset\{z \in \mathbb{C}:|\arg z|<\pi-\varphi<\pi / 2\} \cup\{0\} .
$$

Thus, inequality $(3.15)$ implies that

$$
c_{0}\|u\|_{+}^{2} \leqslant \operatorname{Re}\left\{\exp \left(i \theta_{m}\right) \mathcal{B}_{\lambda ; m}[u, u]\right\} \quad \lambda \in S, \quad|\lambda| \geqslant \sigma_{0},
$$

for all $u \in \stackrel{\circ}{H}_{+}$. Hereinafter $\sigma_{0}$ is some positive number and $\theta_{m}=\theta\left(x_{m}\right), m=1,2, \ldots$

Proceeding as in the proof of inequality $(3.10)$, we find that

$$
\left|\mathcal{B}_{\lambda ; m}[u, v]\right| \leqslant M_{6}\left(M_{4}+|\lambda|\right)\|u\|_{+} \cdot\|v\|_{+}, \quad u, v \in \stackrel{\circ}{H}_{+} .
$$

Inequalities (3.16), (3.17) allow us to apply the generalized Lax-Milgram theorem [1, Thm 2.0.1]. According this theorem, there exists an operator

$$
\mathcal{R}_{m}(\lambda): \stackrel{\circ}{H}_{-} \rightarrow \stackrel{\circ}{H}_{+}
$$

such that

$$
\exp \left(i \theta_{m}\right) \mathcal{B}_{\lambda ; m}\left[\mathcal{R}_{m}(\lambda) F, v\right]=\langle F, v\rangle
$$

for all $F \in \stackrel{\circ}{H}_{-}$and all $v \in \stackrel{\circ}{H}_{+}$;

$$
\left\|\mathcal{R}_{m}(\lambda) F\right\|_{+} \leqslant M_{7}\|F\|_{-}
$$

for all $F \in \stackrel{\circ}{H}_{-}$. Here a number $M_{7}$ is independent of $F$ and $\lambda \in S,|\lambda| \geqslant \sigma_{0}$.

The operator of multiplication by a function $\varphi_{m}$ is denoted by the same symbol. We introduce one more operator

$$
\mathcal{R}(\lambda)=\sum_{m=1}^{\infty} \exp \left(i \theta_{m}\right) \varphi_{m} \mathcal{R}_{m}(\lambda) \varphi_{m}
$$

acting from $\stackrel{\circ}{H}_{-}$into $\stackrel{\circ}{H}_{+}$. 
Employing inequality (3.11) and the boundedness of the coefficients $a_{k l},|k|,|l| \leqslant r$, it is easy to confirm that the operator $\mathbb{R}(\lambda)$ defined by the identity

$$
\langle\mathbb{R}(\lambda) F, v\rangle=B_{\lambda}[\mathcal{R}(\lambda) F, v], \quad v \in \stackrel{\circ}{H}_{+},
$$

acts from $\stackrel{\circ}{H}_{-}$into $\stackrel{\circ}{H}_{-}$.

The functions $\varphi_{m}^{2}, m=1,2, \ldots$, form the partition of the unity in $\mathbb{R}^{n}$, see 3.7 . This is why for all $F \in H_{0}$ and all $v \in \stackrel{\circ}{H}_{+}$, the following identities hold:

$$
\langle F, v\rangle=(F, v)_{\delta}=\sum_{m=1}^{\infty} \int \varphi_{m}^{2}(x) d^{2 \delta}(x) F(x) \overline{v(x)} d x=\sum_{m=1}^{\infty}\left(\varphi_{m} F, \varphi_{m} v\right)_{\delta}
$$

We recall that $(\cdot, \cdot)_{\delta}$ is a scalar product in $H_{0}=L_{2, \delta}\left(\mathbb{R}^{n}\right)$ and as above, all integrals are taken over $\mathbb{R}^{n}$.

Since $a_{k l m}(x)=\left(1-\eta_{m}(x)\right) a_{k l}\left(x_{m}\right)+\eta_{m}(x) a_{k l}(x)$, and the function $\eta_{m}$ is identically equal to one in some neighbourhood of the set $\operatorname{supp} \varphi_{m}$, the functions $a_{k l m}$ and $a_{k l}$ coincide on the set $\operatorname{supp} \varphi_{m}$. This is why it follows from identities 3.2$),(3.20)$ and $(3.21)$ that

$$
\begin{aligned}
\langle\mathbb{R}(\lambda) F, v\rangle= & \sum_{m=1}^{\infty} \exp \left(i \theta_{m}\right)\left\{\sum_{|k|,|l| \leqslant r}\left(d^{-|k|} a_{k l m} D^{k}\left(\varphi_{m} \mathcal{R}_{m}(\lambda) \varphi_{m} F\right), d^{-|l|} v^{(l)}\right)_{\alpha+r}+\right. \\
& \left.+\lambda\left(\mathcal{R}_{m}(\lambda) \varphi_{m} F, \varphi_{m} v\right)_{\delta}\right\} .
\end{aligned}
$$

Hereinafter the symbol $D^{k}$ denotes the differentiation with a multi-index $k$.

Let $F \in H_{0}$. In identity (3.18), we replace $F$ by $\varphi_{m} F$ and $v$ is replaced by $\varphi_{m} v$ :

$$
\exp \left(i \theta_{m}\right) \mathcal{B}_{\lambda ; m}\left[\mathcal{R}_{m}(\lambda) \varphi_{m} F, \varphi_{m} v\right]=\left(\varphi_{m} F, \varphi_{m} v\right)_{\delta}
$$

In view of identity 3.14 this implies that

$$
\begin{aligned}
\left(\varphi_{m} F, \varphi_{m} v\right)_{\delta}= & \exp \left(i \theta_{m}\right)\left\{\sum_{|k|,|l| \leqslant r}\left(d^{-|k|} a_{k l m} D^{k}\left(\mathcal{R}_{m}(\lambda) \varphi_{m} F\right), d^{-|l|} D^{l}\left(\varphi_{m} v\right)\right)_{\alpha+r}\right. \\
& \left.+\lambda\left(\mathcal{R}_{m}(\lambda) \varphi_{m} F, \varphi_{m} v\right)_{\delta}\right\} .
\end{aligned}
$$

Summing up this identity over $m$ from 1 to $\infty$, by 3.22 we have:

$$
\begin{aligned}
\langle F, v\rangle=(F, v)_{\delta}= & \sum_{m=1}^{\infty} \exp \left(i \theta_{m}\right)\left\{\sum_{|k|,|l| \leqslant r}\left(d^{-|k|} a_{k l m} D^{k}\left(\mathcal{R}_{m}(\lambda) \varphi_{m} F\right), d^{-|l|} D^{l}\left(\varphi_{m} v\right)\right)_{\alpha+r}\right. \\
& \left.+\lambda\left(\mathcal{R}_{m}(\lambda) \varphi_{m} F, \varphi_{m} v\right)_{\delta}\right\} .
\end{aligned}
$$

By 3.23 this yields:

$$
\begin{aligned}
\langle\mathbb{R}(\lambda) F, v\rangle-\langle F, v\rangle= & \sum_{m=1}^{\infty} \exp \left(i \theta_{m}\right) \sum_{|k|,|l| \leqslant r}\left\{\left(d^{-|k|} a_{k l m} D^{k}\left(\varphi_{m} \mathcal{R}_{m}(\lambda) \varphi_{m} F\right), d^{-|l|} v^{(l)}\right)_{\alpha+r}\right. \\
& \left.-\left(d^{-|k|} a_{k l m} D^{k}\left(\mathcal{R}_{m}(\lambda) \varphi_{m} F\right), d^{-|l|} D^{l}\left(\varphi_{m} v\right)\right)_{\alpha+r}\right\} .
\end{aligned}
$$

We introduce the notation

$$
U_{m, \lambda}=\mathcal{R}_{m}(\lambda) \varphi_{m} F, \quad m=1,2, \ldots
$$


and we write $(3.24)$ as

$$
\langle\mathbb{R}(\lambda) F, v\rangle-\langle F, v\rangle=\mathbb{K}_{\lambda}[F, v]+\mathbb{L}_{\lambda}[F, v]
$$

where

$$
\begin{aligned}
& \mathbb{K}_{\lambda}[F, v]=\sum_{m=1}^{\infty} \exp \left(i \theta_{m}\right) \sum^{(1)} C_{k^{\prime}}^{k^{\prime \prime}}\left(d^{-|k|} a_{k l m} \varphi_{m}^{\left(k^{\prime}\right)} U_{m, \delta, \lambda}^{\left(k^{\prime \prime}\right)}, d^{-|l|} v^{(l)}\right)_{\alpha+r} \\
& \mathbb{L}_{\lambda}[F, v]=\sum_{m=1}^{\infty} \exp \left(i \theta_{m}\right) \sum^{(2)} C_{l^{\prime}}^{l^{\prime \prime}}\left(d^{-|k|} a_{k l m} U_{m, \delta, \lambda}^{(k)}, d^{-|l|} \varphi_{m}^{\left(l^{\prime}\right)} v^{\left(l^{\prime \prime}\right)}\right)_{\alpha+r}
\end{aligned}
$$

Hereinafter the symbol $\sum^{(1)}$ denotes the summation over the multi-indices $k, l, k^{\prime}, k^{\prime \prime}$ such that $k=k^{\prime}+k^{\prime \prime}, k^{\prime} \neq 0,|k|,|l| \leqslant r$, while the symbol $\sum^{(2)}$ denotes the summation over the multi-indices $k, l, l^{\prime}, l^{\prime \prime}$ such that $l=l^{\prime}+l^{\prime \prime}, l^{\prime} \neq 0,|k|,|l| \leqslant r$.

We are going to estimate the absolute values of the right hand sides in (3.27), (3.28). First let us prove that for all $F \in \stackrel{\circ}{H}_{-}, v \in \stackrel{\circ}{H}_{+}$the inequality holds:

$$
\left|\mathbb{K}_{\lambda}[F, v]\right| \leqslant \omega_{1}(|\lambda|)\|F\|_{-} \cdot\|v\|_{+}, \quad \lambda \in S, \quad|\lambda| \geqslant \sigma_{0},
$$

where a positive function $\omega_{1}(t), t>0$, is such that $\omega_{1}(t) \rightarrow 0$ as $t \rightarrow \infty$.

We consider a symmetric form

$$
\widetilde{\mathcal{B}}_{\lambda ; m}[u, v]=\frac{1}{2}\left\{\exp \left(i \theta_{m}\right) \mathcal{B}_{\lambda m}[u, v]+\exp \left(-i \theta_{m}\right) \overline{\mathcal{B}_{\lambda ; m}[v, u]}\right\},
$$

It follows from 3.16 that

$$
c_{0}\|u\|_{+}^{2} \leqslant \widetilde{\mathcal{B}}_{\lambda ; m}[u, u], \quad u \in \stackrel{\circ}{H}_{+} .
$$

Hence,

$$
c_{0}\|u\|_{\delta}^{2} \leqslant \widetilde{\mathcal{B}}_{\lambda ; m}[u, u], \quad u \in \stackrel{\circ}{H}_{+} .
$$

Then by a well-known theorem in the functional analysis, see, for instance, [26], there exists a self-adjoint operator $B_{m}(\lambda)$ acting in the space $H_{0}=L_{2, \delta}\left(\mathbb{R}^{n}\right)$ such that

$$
\begin{gathered}
\left\|B_{m}^{\frac{1}{2}}(\lambda) u\right\|_{\delta}^{2}=\left(B_{m}^{\frac{1}{2}}(\lambda) u, B_{m}^{\frac{1}{2}}(\lambda) u\right)_{\delta}=\widetilde{\mathcal{B}}_{\lambda ; m}[u, u]=\operatorname{Re}\left\{\exp \left(i \theta_{m}\right) \mathcal{B}_{\lambda ; m}[u, u]\right\} \\
D\left(\widetilde{\mathcal{B}}_{\lambda ; m}\right)=\stackrel{\circ}{H}_{+} .
\end{gathered}
$$

By 3.32 this implies that

$$
\left\|B_{m}^{\frac{1}{2}}(\lambda) u\right\|_{\delta} \geqslant c_{0}\|u\|_{+}, \quad \lambda \in S, \quad|\lambda| \geqslant \sigma_{0}, \quad u \in \stackrel{\circ}{H}_{+} .
$$

Employing (3.1), we get

$$
\left\|d^{\alpha+r-|k|} u^{(k)}\right\|_{0} \leqslant M_{8}\left\|B_{m}^{\frac{1}{2}}(\lambda) u\right\|_{\delta}, \quad|k| \leqslant r .
$$

Therefore, as $\lambda \in S,|\lambda| \geqslant \sigma_{0},|k| \leqslant r$, the operators $d^{\alpha+r-|k|-\delta} D^{k} B_{m}^{-\frac{1}{2}}(\lambda)$ are bounded:

$$
\left\|d^{\alpha+r-|k|} D^{k} B_{m}^{-\frac{1}{2}}(\lambda) u\right\|_{0} \leqslant M_{8}\|u\|_{\delta}
$$

Let multi-indices $k, k^{\prime \prime}$ be such that $0 \neq\left|k^{\prime \prime}\right|<|k| \leqslant r$. As $k^{\prime}=k-k^{\prime \prime}$, we employ (3.8) to obtain

$$
\left\|d^{\alpha+r-|k|} \varphi_{m}^{\left(k^{\prime}\right)} u^{\left(k^{\prime \prime}\right)}\right\|_{0} \leqslant C_{1}\left\|d^{\alpha+r-\left|k^{\prime \prime}\right|} u^{\left(k^{\prime \prime}\right)}\right\|_{0} .
$$

Since $\left|k^{\prime \prime}\right|<|k| \leqslant r$ and $\delta \leqslant r+\alpha$, by means of Lemma 2.1 we prove that

$$
\left\|d^{\alpha+r-\left|k^{\prime \prime}\right|} u^{\left(k^{\prime \prime}\right)}\right\|_{0}<\varepsilon\|u\|_{+}+K(\varepsilon)\|u\|_{\delta}, \quad u \in \stackrel{\circ}{H}_{+} .
$$


Here $\varepsilon>0$ is a sufficiently small positive number and a quantity $K(\varepsilon)$ is such that

$$
K(\varepsilon) \rightarrow+\infty \text { as } \varepsilon \rightarrow+0 .
$$

In the following proof, instead of $M K(\varepsilon)$, where $M$ is some positive number, we shall write again $K(\varepsilon)$.

By $(3.34)$, it follows from 3.36 that

$$
\left\|d^{\alpha+r-\left|k^{\prime \prime}\right|} u^{\left(k^{\prime \prime}\right)}\right\|_{0}^{2}<\varepsilon^{2}\left\|B_{m}^{\frac{1}{2}}(\lambda) u\right\|_{\delta}^{2}+K(\varepsilon)^{2}\|u\|_{\delta}^{2} .
$$

In view of identity 3.33 we have

$$
\left\|d^{\alpha+r-\left|k^{\prime \prime}\right|} u^{\left(k^{\prime \prime}\right)}\right\|_{0}^{2} \leqslant \varepsilon^{2} \operatorname{Re}\left\{\exp \left(i \theta_{m}\right) \mathcal{B}_{\lambda ; m}[u, u]\right\}+K(\varepsilon)^{2}\|u\|_{\delta}^{2} .
$$

Employing (3.14), we estimate the right hand side of this inequality:

$$
\begin{aligned}
& \varepsilon^{2} \operatorname{Re}\left\{\exp \left(i \theta_{m}\right) \mathcal{B}_{\lambda ; m}[u, u]\right\}+K(\varepsilon)^{2}\left\|d^{\delta} u\right\|_{0}^{2} \\
& =\varepsilon^{2} \operatorname{Re}\left(\exp \left(i \theta_{m}\right)\left(\sum_{|k|,|l| \leqslant r}\left(d^{-|k|} a_{k l m} u^{(k)}, d^{-|l|} u^{(l)}\right)_{\alpha+r}+\lambda\left\|d^{\delta} u\right\|_{0}^{2}\right)\right)+K(\varepsilon)^{2}\|u\|_{\delta}^{2} \\
& \quad \leqslant \varepsilon^{2} \operatorname{Re}\left(\exp \left(i \theta_{m}\right)\left(\sum_{|k|,|l| \leqslant r}\left(d^{-|k|} a_{k l m} u^{(k)}, d^{-|l|} u^{(l)}\right)_{\alpha+r}+\Lambda(|\lambda|, \varepsilon)\|u\|_{\delta}^{2}\right)\right),
\end{aligned}
$$

where $\Lambda(|\lambda|, \varepsilon)$ is a continuous positive function satisfying the condition

$$
|\lambda|+K(\varepsilon)^{2} \varepsilon^{-2} \leqslant \cos (\varphi-\pi / 2) \Lambda(|\lambda|, \varepsilon) .
$$

By (3.37), without loss of generality we can assume that $K(\varepsilon)^{2} \varepsilon^{-2} \rightarrow+\infty$ as $\varepsilon \rightarrow+0$. Hence, the above obtained inequality with $|\lambda|=1 / \varepsilon$ implies that

$$
\begin{aligned}
& \left\|d^{\alpha+r-\left|k^{\prime \prime}\right|} u^{\left(k^{\prime \prime}\right)}\right\|_{0}^{2} \\
& \leqslant \varepsilon^{2} \operatorname{Re}\left(\exp \left(i \theta_{m}\right)\left(\sum_{|k|,|l| \leqslant r}\left(d^{-|k|} a_{k l m} u^{(k)}, d^{-|l|} u^{(l)}\right)_{\alpha+r}+p(\varepsilon)\|u\|_{\delta}^{2}\right)\right),
\end{aligned}
$$

where $p(\varepsilon)=\Lambda(1 / \varepsilon, \varepsilon)$. We note that $p(\varepsilon) \rightarrow \infty$ as $\varepsilon \rightarrow 0$. We denote by $q(\cdot)$ the inverse function for $p(\varepsilon)$. Then as $\varepsilon=q(|\lambda|)$, that is, $|\lambda|=p(\varepsilon)$, it follows from (3.38) that

$$
\left\|d^{\alpha+r-\left|k^{\prime \prime}\right|} u^{\left(k^{\prime \prime}\right)}\right\|_{0}^{2} \leqslant q(\lambda)^{2} \operatorname{Re}\left(\exp \left(i \theta_{m}\right)\left(\sum_{|k|,|l| \leqslant r}\left(d^{-|k|} a_{k l m} u^{(k)}, d^{-|l|} u^{(l)}\right)_{\alpha+r}+\lambda\|u\|_{\delta}^{2}\right)\right) .
$$

Here $q(t)$ is a positive continuous function defined for $t>0$ such that $q(t) \rightarrow 0$ as $t \rightarrow \infty$. By (3.14), 3.33) and the above obtained inequality we obtain that

$$
\left\|d^{\alpha+r-\left|k^{\prime \prime}\right|} u^{\left(k^{\prime \prime}\right)}\right\|_{0} \leqslant q(|\lambda|)\left\|B_{m}^{\frac{1}{2}}(\lambda) u\right\|_{\delta}, \quad u \in \stackrel{\circ}{H}_{+} .
$$

We have proved this inequality for $0 \neq\left|k^{\prime \prime}\right|<|k|$. In what follows we shall prove that it holds also in the case $\left|k^{\prime \prime}\right|=0$.

Let $\lambda \in S$ and $|\lambda|>\sigma_{0}$, where $\sigma_{0}>0$ is the same number as in (3.34). Then employing (3.30) we get

$$
\begin{aligned}
\left\|B_{m}^{\frac{1}{2}}(\lambda) u\right\|_{\delta}^{2} & =\operatorname{Re}\left\{\exp \left(i \theta_{m}\right) \mathcal{B}_{\lambda ; m}[u, u]\right\}=\left\|B_{m}^{\frac{1}{2}}\left(\lambda_{0}\right) u\right\|_{\delta}^{2}+\operatorname{Re}\left\{\exp \left(i \theta_{m}\right)\left(\lambda-\sigma_{0}\right)\right\}\|u\|_{\delta}^{2} \\
& \geqslant \operatorname{Re}\left\{\exp \left(i \theta_{m}\right)\left(\lambda-\sigma_{0}\right)\right\}\|u\|_{\delta}^{2}
\end{aligned}
$$


Therefore,

Denoting

$$
\|u\|_{\delta}^{2} \leqslant \frac{1}{\operatorname{Re}\left\{\exp \left(i \theta_{m}\right)\left(\lambda-\sigma_{0}\right)\right\}}\left\|B_{m}^{\frac{1}{2}}(\lambda) u\right\|_{\delta}^{2}
$$

$$
q(\lambda)=\frac{1}{\sqrt{\operatorname{Re}\left\{\exp \left(i \theta_{m}\right)\left(\lambda-\sigma_{0}\right)\right\}}}
$$

and taking into consideration the inequality $d^{r+\alpha}(x) \leqslant d^{\delta}(x), x \in \mathbb{R}^{n}$, by 3.40 we arrive at (3.39) for $\left|k^{\prime \prime}\right|=0$.

According inequalities (3.16), (3.17), the form $\exp \left(i \theta_{m}\right) \mathcal{B}_{\lambda ; m}[u, v]$ is closed and sectorial in the space $H_{0}$. This is why, by Statement i) of Theorem 2.1 in [27, Ch. 6], there exists an $m$-sectorial operator $A_{m}(\lambda)$ such that

$$
\exp \left(i \theta_{m}\right) \mathcal{B}_{\lambda ; m}[u, v]=\left(A_{m}(\lambda) u, v\right)_{\delta}, \quad u \in D\left(A_{m}(\lambda)\right) \subset \stackrel{\circ}{H}_{+}, \quad v \in \stackrel{\circ}{H}_{+} .
$$

Let $f \in H_{0}$. Then $\mathcal{R}_{m}(\lambda) f \in \stackrel{\circ}{H}_{+}$and in view of identity 3.18 we have:

$$
\exp \left(i \theta_{m}\right) \mathcal{B}_{\lambda ; m}\left[\mathcal{R}_{m}(\lambda) f, v\right]=\langle f, v\rangle=(f, v)_{\delta}
$$

for all $v \in \stackrel{\circ}{H}_{+}$. According Statement iii) of Theorem 2.1 in [27, Ch. 6], if for $u \in \stackrel{\circ}{H}_{+}, w \in H_{0}$ the identity holds

$$
\exp \left(i \theta_{m}\right) \mathcal{B}_{\lambda ; m}[u, v]=(w, v)_{\delta} \quad \forall v \in \stackrel{\circ}{H}_{+},
$$

then $u \in D\left(A_{m}(\lambda)\right)$ and $A_{m}(\lambda) u=w$. This is why

$$
\left(A_{m}(\lambda) \mathcal{R}_{m}(\lambda) f, v\right)_{\delta}=\langle f, v\rangle=(f, v)_{\delta} \quad \forall v \in \stackrel{\circ}{H}_{+} .
$$

This yields that

$$
A_{m}(\lambda) \mathcal{R}_{m}(\lambda) f=f \quad \text { for all } \quad f \in H_{0}
$$

and therefore,

$$
\mathcal{R}_{m}(\lambda) f=A_{m}^{-1}(\lambda) f \quad \text { for all } \quad f \in H_{0} .
$$

Let $B_{m}(\lambda)$ be a self-adjoint operator associated with form 3.30 . It follows from inequality (3.34 that

$$
\left\|B_{m}^{\frac{1}{2}}(\lambda) u\right\|_{\delta} \geqslant c_{0}\|u\|_{\delta} \quad \text { for all } \quad u \in \stackrel{\circ}{H}_{+}, \quad \lambda \in S, \quad|\lambda| \geqslant \sigma_{0}
$$

This implies the invertibility of the operator $B_{m}^{\frac{1}{2}}(\lambda)$ as $\lambda \in S,|\lambda| \geqslant \sigma_{0}$. Applying Theorem 3.2 from [27, Ch. 6], we obtain the representation

$$
A_{m}^{-1}(\lambda)=B_{m}^{-\frac{1}{2}}(\lambda) X_{m}(\lambda) B_{m}^{-\frac{1}{2}}(\lambda), \quad \lambda \in S, \quad|\lambda| \geqslant \sigma_{0}
$$

where $X_{m}(\lambda)$ is some bounded operator in $H_{0}$ and its norm $\left\|X_{m}(\lambda)\right\|$ does not exceed a number $M_{1}>0$ independent of $\lambda \in S,|\lambda| \geqslant \sigma_{0}$.

We proceed to proving estimate 3.29$)$. We rewrite identity (3.27) as

$$
\mathbb{K}_{\lambda}[F, v]=\sum_{m=1}^{\infty} \exp \left(i \theta_{m}\right) \sum^{(1)} C_{k^{\prime}}^{k^{\prime \prime}}\left(d^{-|k|} a_{k l m} \varphi_{m}^{\left(k^{\prime}\right)} U_{m, \lambda}^{\left(k^{\prime \prime}\right)}, d^{-|l|} v^{(l)}\right)_{\alpha+r} .
$$

Let $F \in H_{0}$. Employing identities (3.41)-(3.44), we get:

$$
\begin{aligned}
\mathbb{K}_{\lambda}[F, v] & =\sum_{m=1}^{\infty} \sum^{(1)} C_{k^{\prime}}^{k^{\prime \prime}} \exp \left(i \theta_{m}\right)\left(d^{-|k|} a_{k l m} \varphi_{m}^{\left(k^{\prime}\right)} D^{k^{\prime \prime}} A_{m}^{-1}(\lambda) \varphi_{m} F, d^{-|l|} v^{(l)}\right)_{\alpha+r} \\
& =\sum_{m=1}^{\infty} \sum^{(1)} C_{k^{\prime}}^{k^{\prime \prime}} \exp \left(i \theta_{m}\right)\left(d^{-|k|} a_{k l m} \varphi_{m}^{\left(k^{\prime}\right)} D^{k^{\prime \prime}} B_{m}^{-\frac{1}{2}}(\lambda) X_{m}(\lambda) B_{m}^{-\frac{1}{2}}(\lambda) \varphi_{m} F, d^{-|l|} v^{(l)}(x)\right)_{\alpha+r}
\end{aligned}
$$


Employing Lemma 3.2 and Cauchy-Bunyakovsky inequality, we obtain:

$$
\left|\mathbb{K}_{\lambda}[F, v]\right| \leqslant M_{9} \Lambda_{n} \sup _{m=1,2, \ldots} \sum^{(3)}\left\|\mathbb{T}_{m}^{k^{\prime}, k^{\prime \prime}}(\lambda) V_{m, \lambda}\right\|_{r+\alpha} \cdot\left\|d^{-|l|} v^{(l)}(x)\right\|_{r+\alpha},
$$

where

$$
\mathbb{T}_{m}^{k^{\prime}, k^{\prime \prime}}(\lambda)=d^{-\left|k^{\prime}\right|-\left|k^{\prime \prime}\right|} \varphi_{m}^{\left(k^{\prime}\right)} a_{k l m} D^{k^{\prime \prime}} B_{m}^{-\frac{1}{2}}(\lambda), \quad V_{m, \lambda}=X_{m}(\lambda) B_{m}^{-\frac{1}{2}}(\lambda) \varphi_{m} F
$$

and the symbol $\sum^{(3)}$ denotes the summation over multi-indices $k^{\prime}, k^{\prime \prime}$ such that $\left|k^{\prime}\right|+\left|k^{\prime \prime}\right| \leqslant r$ and $k^{\prime} \neq 0$.

By inequality (3.1), it follows from (3.45) that

$$
\left|\mathbb{K}_{\lambda}[F, v]\right| \leqslant M_{10}\|v\|_{+} \cdot \sup _{m=1,2, \ldots} \sum^{(3)}\left\|\mathbb{T}_{m}^{k^{\prime}, k^{\prime \prime}}(\lambda) V_{m, \lambda}\right\|_{r+\alpha},
$$

Let $\sigma_{0}$ be the same positive number as in $(3.43)$. Then for $|\lambda|>\sigma_{0}$, by identity $(3.33)$ we have

$$
\left\|B_{m}^{\frac{1}{2}}(\lambda) u\right\|_{\delta}^{2}=\operatorname{Re}\left\{\exp \left(i \theta_{m}\right) \mathcal{B}_{\lambda ; m}[u, u]\right\} \geqslant \operatorname{Re}\left\{\exp \left(i \theta_{m}\right) \mathcal{B}_{\sigma_{0} ; m}[u, u]\right\}=\left\|B_{m}^{\frac{1}{2}}\left(\sigma_{0}\right) u\right\|_{\delta}^{2} .
$$

Therefore,

$$
\left\|B_{m}^{-\frac{1}{2}}(\lambda) \varphi_{m} F\right\|_{\delta} \leqslant M_{11}\left\|B_{m}^{-\frac{1}{2}}\left(\sigma_{0}\right) \varphi_{m} F\right\|_{\delta} .
$$

In what follows we shall make us of the identity:

$$
\|f\|_{\delta}=\sup \left|(f, v)_{\delta}\right|,
$$

where the supremum is taken over all $v \in C_{0}^{\infty}\left(\mathbb{R}^{n}\right)$, such that $\|v\|_{\delta}=1$.

As $\lambda=\sigma_{0}$, by identity 3.33 we have:

$$
\left(B_{m}^{\frac{1}{2}}\left(\sigma_{0}\right) u, B_{m}^{\frac{1}{2}}\left(\sigma_{0}\right) v\right)_{\delta}=\widetilde{\mathcal{B}}_{\sigma_{0} ; m}[u, v] .
$$

On the other hand,

$$
\begin{aligned}
& \widetilde{\mathcal{B}}_{\sigma_{0} ; m}[u, u] \geqslant c_{1}\|u\|_{+}^{2}, \\
& \left|\widetilde{\mathcal{B}}_{\sigma_{0} ; m}[u, v]\right| \leqslant M_{1}\left(M_{0}+\sigma_{0}\right)\|u\|_{+} \cdot\|v\|_{+}
\end{aligned}
$$

for all $u, v \in \stackrel{\circ}{H}_{+}$. Hence, according Lax-Milgram theorem, equation

$$
\widetilde{\mathcal{B}}_{\sigma_{0} ; m}[u, \widehat{v}]=(w, \widehat{v})_{\delta} \quad \text { for all } \quad \widehat{v} \in C_{0}^{\infty}\left(\mathbb{R}^{n}\right)
$$

is solvable for each $w \in H_{0}=L_{2, \delta}\left(\mathbb{R}^{n}\right)$. Therefore, the function $v \in C_{0}^{\infty}\left(\mathbb{R}^{n}\right)$ in 3.50$)$ can be represented as $v=B_{m}^{\frac{1}{2}}\left(\sigma_{0}\right) w$, that is,

$$
\|f\|_{\delta}=\sup \left|\left(f, B_{m}^{\frac{1}{2}}\left(\sigma_{0}\right) w\right)_{\delta}\right|
$$

where the supremum is taken over all $w \in C_{0}^{\infty}\left(\mathbb{R}^{n}\right)$ such that $\left\|B_{m}^{\frac{1}{2}}\left(\sigma_{0}\right) w\right\|_{\delta}=1$.

On the other hand, on the class $C_{0}^{\infty}\left(\mathbb{R}^{n}\right)$, the norms $\|v\|_{+}$and $\left\|B_{m}^{\frac{1}{2}}\left(\sigma_{0}\right) v\right\|_{\delta}$ are equivalent. This is why

$$
\begin{aligned}
\left\|B_{m}^{-\frac{1}{2}}\left(\sigma_{0}\right) \varphi_{m} F\right\|_{\delta} & =\sup \left|\left(B_{m}^{-\frac{1}{2}}\left(\sigma_{0}\right) \varphi_{m} F, w\right)_{\delta}\right| \\
& =\sup \left|\left(B_{m}^{-\frac{1}{2}}\left(\sigma_{0}\right) \varphi_{m} F, B_{m}^{\frac{1}{2}}\left(\sigma_{0}\right) v\right)_{\delta}\right| \\
& \leqslant M_{12} \sup \left|\left(\varphi_{m} F, v\right)_{\delta}\right| \leqslant M_{13}\left\|\varphi_{m} F\right\|_{-} \leqslant M_{14}\|F\|_{-},
\end{aligned}
$$


where the first supremum in this chain is taken over all $w \in C_{0}^{\infty}\left(\mathbb{R}^{n}\right)$ with the unit norm in $H_{0}$, the second supremum is taken over all $v \in C_{0}^{\infty}\left(\mathbb{R}^{n}\right)$ obeying the condition $\left\|B_{m}^{\frac{1}{2}}\left(\sigma_{0}\right) v\right\|_{\delta}=1$, while the third supremum is taken over all $v \in C_{0}^{\infty}\left(\mathbb{R}^{n}\right)$ with the unit norm in $H_{+}$.

By (3.49), (3.52) we have the inequality

$$
\left\|V_{m, \lambda}\right\|_{\delta} \leqslant M\|F\|_{-},
$$

which is valid as $\lambda \in S,|\lambda| \geqslant \sigma_{0}$, where $\sigma_{0} \geqslant 1$ is some finite number.

By the above proven inequality (3.39), it follows from identity $(3.46)$ that

$$
\lim _{\lambda \rightarrow \infty}\left\|\mathbb{T}_{m}^{k^{\prime}, k^{\prime \prime}}(\lambda)\right\|=0 .
$$

Then by 3.47$),(3.52),(3.53)$ we get

$$
\left|\mathbb{K}_{\lambda}[F, v]\right| \leqslant M_{15} \sup _{m=1,2, \ldots\left|k^{\prime}\right|+\left|k^{\prime \prime}\right| \leqslant 2 r ; k^{\prime} \neq 0}\left\|\mathbb{T}_{m}^{k^{\prime}, k^{\prime \prime}}(\lambda)\right\|\left\|V_{m, \lambda}\right\|_{\delta}\|v\|_{+} \leqslant \omega_{1}(|\lambda|)\|F\|_{-}\|v\|_{+}
$$

for all $F \in H_{0}, v \in \stackrel{\circ}{H}_{+}$, and $\omega_{1}(t) \rightarrow 0$ as $t \rightarrow \infty$. And since $H_{0}$ is dense in $\stackrel{\circ}{H}_{-}$, this implies estimate $(3.29)$.

We proceed to estimating the absolute value of the right hand side in the identity 3.30 . Let us prove that for all $F \in \stackrel{\circ}{H}_{-}, v \in \stackrel{\circ}{H}_{+}$, the inequality holds:

$$
\left|\mathbb{L}_{\lambda}[F, v]\right| \leqslant \omega_{2}\left(\sigma_{0}\right)\|F\|_{-} \cdot\|v\|_{+}, \quad \lambda \in S, \quad|\lambda| \geqslant \sigma_{0},
$$

where a positive function $\omega_{2}(t), t>\sigma_{0}$, is such that $\omega_{2}(t) \rightarrow 0$ as $t \rightarrow \infty$.

We represent the sesquilinear form $\mathbb{L}_{\lambda}[F, v]$, see $(3.28)$, as

$$
\mathbb{L}_{\lambda}[F, v]=\sum_{m=1}^{\infty} \exp \left(i \theta_{m}\right) \sum^{(2)} C_{l^{\prime}}^{l^{\prime \prime}} \mathbb{I}_{\lambda ; k, m}^{l^{\prime}, l^{\prime \prime}}[F, v],
$$

where

$$
\begin{aligned}
& \mathbb{I}_{\lambda ; k, m}^{l^{\prime}, l^{\prime \prime}}[F, v]=\left(d^{-|k|} a_{k l m} U_{m, \lambda}^{(k)}, d^{-\left|l^{\prime}\right|-\left|l^{\prime \prime}\right|} \varphi_{m}^{\left(l^{\prime}\right)} v^{\left(l^{\prime \prime}\right)}\right)_{\alpha+r}, \\
& U_{m, \lambda}^{(k)}(x)=D^{k}\left(\mathcal{R}_{m}(\lambda) \varphi_{m} F\right)(x), \quad m=1,2, \ldots,
\end{aligned}
$$

and the symbol $\sum^{(2)}$ denotes the summation over the multi-indices $k, l, l^{\prime}, l^{\prime \prime}$ such that $l=l^{\prime}+l^{\prime \prime}$, $l^{\prime} \neq 0,|k|,|l| \leqslant r$.

Employing 3.42$), 3.43), 3.46$, we write the form $\mathbb{I}_{\lambda ; k, m}^{l^{\prime}, l^{\prime \prime}}[F, v]$ as

$$
\mathbb{I}_{\lambda ; k, m}^{l^{\prime}, l^{\prime \prime}}[F, v]=\left(d^{-|k|} a_{k l m} D^{k} B_{m}^{-\frac{1}{2}}(\lambda) V_{m, \lambda}, d^{-\left|l^{\prime}\right|-\left|l^{\prime \prime}\right|} \varphi_{m}^{\left(l^{\prime}\right)} D^{l^{\prime \prime}} v\right)_{\alpha+r} .
$$

Hence, in view of the self-adjointness of the operator $B_{m}\left(\sigma_{0}\right)$, we get:

$$
\mathbb{I}_{\lambda ; k, m}^{l^{\prime} l^{\prime \prime}}[F, v]=\left(B_{m}^{-\frac{1}{2}}\left(\sigma_{0}\right) D^{l^{\prime \prime}} \varphi_{m}^{\left(l^{\prime}\right)} d^{-|k|-\left|l^{\prime}\right|-\left|l^{\prime \prime}\right|} a_{k l m} D^{k} B_{m}^{-\frac{1}{2}}(\lambda) V_{m, \lambda}, B_{m}^{\frac{1}{2}}\left(\sigma_{0}\right) v\right)_{r+\alpha} .
$$

According (3.46) and (3.54),

$$
\begin{aligned}
& \left(\mathbb{T}_{m}^{l^{\prime}, l^{\prime \prime}}\left(\sigma_{0}\right)\right)^{*}=B_{m}^{-\frac{1}{2}}\left(\sigma_{0}\right) D^{l^{\prime \prime}} \varphi_{m}^{\left(l^{\prime}\right)} d^{-\left|l^{\prime}\right|-\left|l^{\prime \prime}\right|} a_{k l m}, \\
& \lim _{\sigma_{0} \rightarrow \infty}\left\|\left(\mathbb{T}_{m}^{l^{\prime}, l^{\prime \prime}}\left(\sigma_{0}\right)\right)^{*}\right\|=0
\end{aligned}
$$

as $\left|l^{\prime}\right|+\left|l^{\prime \prime}\right| \leqslant r ; l^{\prime} \neq 0$. Taking this into consideration, we write identity (3.57) as

$$
\mathbb{I}_{\lambda ; k, m}^{l^{\prime}, l^{\prime \prime}}[F, v]=\left(\left(\mathbb{T}_{m}^{l^{\prime}, l^{\prime \prime}}\left(\sigma_{0}\right)\right)^{*} d^{-|k|} D^{k} B_{m}^{-\frac{1}{2}}(\lambda) V_{m, \lambda}, B_{m}^{\frac{1}{2}}\left(\sigma_{0}\right) v\right)_{\alpha+r} .
$$

We introduce the notation

$$
\mathbb{P}_{m, k}\left(\sigma_{0}\right)=d^{-|k|} D^{k} B_{m}^{-\frac{1}{2}}\left(\sigma_{0}\right)
$$


and we write the obtained identity as

$$
\mathbb{I}_{\lambda ; k, m}^{l^{\prime}, l^{\prime \prime}}[F, v]=\left(\left(\mathbb{T}_{m}^{l^{\prime}, l^{\prime \prime}}\left(\sigma_{0}\right)\right)^{*} \mathbb{P}_{m, k}\left(\sigma_{0}\right) B_{m}^{\frac{1}{2}}\left(\sigma_{0}\right) B_{m}^{-\frac{1}{2}}(\lambda) V_{m, \lambda}, B_{m}^{\frac{1}{2}}\left(\sigma_{0}\right) v\right)_{\alpha+r}
$$

It follows 3.48 that as $\lambda \in S,|\lambda| \geqslant \sigma_{0}$, the operator $B_{m}^{\frac{1}{2}}\left(\sigma_{0}\right) B_{m}^{-\frac{1}{2}}(\lambda)$ is a bounded operator and its norm does not exceed one. On the other hand, according inequality (3.35), the operator $\mathbb{P}_{m, k}\left(\sigma_{0}\right)$ is bounded. This is why by $(3.59)$ we have

$$
\left|\mathbb{I}_{\lambda ; k, m}^{l^{\prime}, l^{\prime \prime}}[F, v]\right| \leqslant M_{16}\left\|\left(\mathbb{T}_{m}^{l^{\prime} l^{\prime \prime}}\left(\sigma_{0}\right)\right)^{*}\right\|\left\|V_{m, \lambda}\right\|_{\delta}\left\|B_{m}^{\frac{1}{2}}\left(\sigma_{0}\right) v\right\|_{\delta}
$$

for all $\lambda \in S,|\lambda| \geqslant \sigma_{0}$. By (3.51), (3.53) it follows that

$$
\left|\mathbb{I}_{\lambda ; k, m}^{l^{\prime}, l^{\prime \prime}}[F, v]\right| \leqslant M_{17}\left\|\left(\mathbb{T}_{m}^{l^{\prime}, l^{\prime \prime}}\left(\sigma_{0}\right)\right)^{*}\right\|\|F\|_{-}\|v\|_{+}
$$

for all $\lambda \in S,|\lambda| \geqslant \sigma_{0}$ and for all $F \in H_{0}, v \in \stackrel{\circ}{H}_{+}$. Introducing the notation

we obtain:

$$
\omega_{*}\left(\sigma_{0}\right)=M_{17}\left\|\left(\mathbb{T}_{m}^{l^{\prime}, l^{\prime \prime}}\left(\sigma_{0}\right)\right)^{*}\right\|
$$

$$
\left|\mathbb{I}_{\lambda ; k, m}^{l^{\prime}, l^{\prime \prime}}[F, v]\right| \leqslant \omega_{*}\left(\sigma_{0}\right)\|F\|_{-}\|v\|_{+}
$$

for all $\lambda \in S,|\lambda| \geqslant \sigma_{0}$ and all $F \in H_{0}, v \in \stackrel{\circ}{H}_{+}$.

It follows from $(3.58)$ that $\omega_{*}\left(\sigma_{0}\right) \rightarrow 0$ as $\sigma_{0} \rightarrow 0$. This is why by choosing the number $\sigma_{0}$ large enough, by 3.60$), 3.56$ and Lemma 3.2 we obtain 3.55 for $F \in H_{0}$. By the continuity this estimate is extended to all $F \in \stackrel{\circ}{H}_{-}$. This completes the proof of inequality 3.55 ).

Applying inequalities 3.29$),(3.55)$, by $(3.26)$ we obtain

$$
|\langle\mathbb{R}(\lambda) F, v\rangle-\langle F, v\rangle| \leqslant\left(\omega_{1}(|\lambda|)+\omega_{2}\left(\sigma_{0}\right)\right)\|F\|_{-}\|v\|_{+}
$$

for all $F \in \stackrel{\circ}{H}_{-}, v \in \stackrel{\circ}{H}_{+}$. Since $\lim _{t \rightarrow \infty} \omega_{i}(t)=0, i=1,2$, there exists a number $\sigma_{0} \geqslant 1$ such that

$$
|\langle\mathbb{R}(\lambda) F, v\rangle-\langle F, v\rangle| \leqslant \frac{1}{2}\|F\|_{-}\|v\|_{+}
$$

for each $\lambda \in S,|\lambda| \geqslant \sigma_{0}$ and all $F \in \stackrel{\circ}{H}_{-}, v \in \stackrel{\circ}{H}_{+}$.

It follows from (3.61) estimate that as $\lambda \in S,|\lambda| \geqslant \sigma_{0}$, the operator $\mathbb{G}(\lambda)=\mathbb{R}(\lambda)-E$ acting from $\stackrel{\circ}{H}_{-}$into $\stackrel{\vec{H}}{-}_{-}$is bounded and its norm does not exceed $\frac{1}{2}$. This is why the operator $\mathbb{R}(\lambda): \stackrel{\circ}{H}_{-} \rightarrow \stackrel{\circ}{H}_{-}$is continuously invertible and $\mathbb{R}^{-1}(\lambda)=(E+\mathbb{G}(\lambda))^{-1}$.

The operator $\mathcal{R}_{m}(\lambda)$ defined by identity $(3.18)$ acts from $\stackrel{\circ}{H}_{-}$into $\stackrel{\circ}{H}_{+}$. This is why it follows from 3.20 that the operator $\mathcal{R}(\lambda)$ also acts from $\stackrel{\circ}{H}_{-}$into $\stackrel{\circ}{H}_{+}$. Therefore, for each functional $F \in H_{-}$, the function $U(x)$ defined by the identity

$$
U=\mathcal{R}(\lambda) \mathbb{R}^{-1}(\lambda) F, \quad \lambda \in S, \quad|\lambda| \geqslant \sigma_{0},
$$

belongs to the space $\stackrel{\circ}{H}_{+}$.

In what follows we suppose that $\lambda \in S,|\lambda| \geqslant \sigma_{0}$ and $\sigma_{0}$ is some sufficiently large number. Then it follows from identity $(3.21)$ that $B_{\lambda}\left[\mathcal{R}(\lambda) \mathbb{R}^{-1}(\lambda) F, v\right]=\langle F, v\rangle$ for all $v \in C_{0}^{\infty}\left(\mathbb{R}^{n}\right)$. This is why for $\lambda \in S,|\lambda| \geqslant \sigma_{0}$, the function $U(x)$ defined by identity $(3.62)$ solves the equation

$$
B_{\lambda}[U, v]=\langle F, v\rangle \text { for all } v \in C_{0}^{\infty}\left(\mathbb{R}^{n}\right),
$$

that is, it is a solution to problem $D_{\lambda}$. Since as $\lambda \in S,|\lambda| \geqslant \sigma_{0}$ the operator $\mathbb{R}^{-1}(\lambda)$ is bounded, it follows from $(3.19)$ and $(3.20)$ that the function $(3.62)$ satisfies estimate $(3.9)$ of Theorem 3.1 .

We proceed to proving the uniqueness of the solution of problem $D_{\lambda}$. It is clear that it is sufficient to prove that homogeneous problem $D_{\lambda}$, that is, as $F=0$, has only the trivial solution. 
We consider the adjoint problem: given a functional $F \in \stackrel{\circ}{H}_{-}$, find a function $U_{1} \in \stackrel{\circ}{H}_{+}$ satisfying the identity

$$
\overline{B_{\lambda}\left[v, U_{1}\right]}=\langle F, v\rangle \quad \text { for all } \quad v \in \stackrel{\circ}{H}_{+} .
$$

Since the coefficients of the form $\overline{B_{\lambda}\left[v, U_{1}\right]}$ satisfy the assumptions of Theorem 3.1, we proceed as above and we construct the operators $\mathcal{R}_{*}(\lambda), \mathbb{R}_{*}(\lambda)$ such that the function

$$
U_{1}=\mathcal{R}_{*}(\lambda) \mathbb{R}_{*}(\lambda)^{-1} F, \quad \lambda \in S, \quad|\lambda| \geqslant \sigma_{0}^{*}
$$

belongs to the space $\stackrel{\circ}{H}_{+}$and satisfies equation 3.63 .

Let a function $u \in \stackrel{\circ}{H}_{+}$solves the equation

$$
B_{\lambda}[u, v]=0 \text { for all } v \in \stackrel{\circ}{H}_{+},
$$

where $\lambda \in S,|\lambda| \geqslant \sigma_{0}^{\prime}=\max \left\{\sigma_{0}^{*}, \sigma_{0}\right\}$. Let $F$ be an arbitrary element in the space $\stackrel{\circ}{H}_{-}$. Since $U_{1}=\mathcal{R}_{*}(\lambda) \mathbb{R}_{*}(\lambda)^{-1} F$ belongs to the space $\stackrel{\circ}{H}_{+}$, we let $v=U_{1}$ in 3.64 and we obtain $B_{\lambda}\left[u, U_{1}\right]=0$, that is, $\overline{B_{\lambda}\left[u, U_{1}\right]}=0$.

On the other hand, the function $U_{1}=\mathcal{R}_{*}(\lambda) \mathbb{R}_{*}(\lambda)^{-1} F$ satisfies $(3.63)$. This is why $\langle F, u\rangle=0$ for all $F \in \stackrel{\circ}{H}_{+}$. In view of the embedding $\stackrel{\circ}{H}_{+} \rightarrow \stackrel{\circ}{H}_{-}$and letting $F=u$, we have $\langle u, u\rangle=0$, that is, $u=0$.

\section{Smoothness of Solution to homogeneous Variational Dirichlet problem}

If the coefficients $a_{k l}$ of form (3.2) and the right hand side in equation (3.3), the functional $F$, possess certain smoothness, the smoothness of the solution of problem $D_{\lambda}$ improves as well.

Let $m$ be a natural number and $m \leqslant r$. We introduce the notations $\stackrel{\circ}{H}_{+}^{m}=\stackrel{\circ}{W}_{2 ; \alpha-m, \delta}^{r+m}\left(\mathbb{R}^{n}\right)$, $\|\cdot\|_{+m}$ is the norm in $W_{2 ; \alpha-m, \delta}^{r+m}\left(\mathbb{R}^{n}\right)$. By $\stackrel{\circ}{H}_{-}^{-m}$ we denote the completion of the space $H_{0}=$ $L_{2, \delta}\left(\mathbb{R}^{n}\right)$ with respect to the norm

$$
\|f\|_{-m}=\sup \left|(f, u)_{\delta}\right|,
$$

where the supremum is taken over all $u \in C_{0}^{\infty}\left(\mathbb{R}^{n}\right)$ such that $\left\|u ; W_{2 ; \alpha+m, \delta}^{r-m}\left(\mathbb{R}^{n}\right)\right\|=1$. We note that $\stackrel{\circ}{H}_{+}^{m} \rightarrow \stackrel{\circ}{H}_{+}, \stackrel{\circ}{H}_{-}^{-m} \rightarrow \stackrel{\circ}{H}_{-}$for each natural $m \leqslant r$.

Theorem 4.1. Let all assumptions of Theorem 3.1 are satisfied and there exists a natural number $m_{0} \leqslant r$ such that

$$
\left|a_{k l}^{(s)}(x)\right| \leqslant M d^{|s|}(x), \quad x \in \mathbb{R}^{n}
$$

for each multi-index $s,|s| \leqslant m_{0}$.

Then there exists a sector $S \subset\{z \in \mathbb{C}:|\arg z|<\pi / 2\} \cup\{0\}$ with the vertex at the origin and a positive number $\sigma_{0}$ such that as $\lambda \in S$ and $|\lambda| \geqslant \sigma_{0}$, for each given $F \in \stackrel{\circ}{H}_{-}^{-m}$, where a natural number $m$ obeys the inequality $m \leqslant m_{0}$, there exists a unique solution $u \in \stackrel{\circ}{H}_{+}$of Problem $D_{\lambda}$. This solution belongs to the space $\stackrel{\circ}{H}_{+}^{m}$ and the estimate

$$
\|u\|_{+m} \leqslant M\|F\|_{-m}
$$

holds, where a number $M>0$ is independent of $\lambda \in S,|\lambda| \geqslant \sigma_{0}$, and the functional $F$.

The proof is based on an improvement of the technique employed in the proof of Theorem 8 of work [12] on the smoothness of a solution to the variational Dirichlet problem in a bounded domain associated with a non-coercive form, see also [28]. 


\section{BIBLIOGRAPHY}

1. S.M. Nikol'skiū, P.I. Lizorkin, N.V. Miroshin. Weighted function spaces and their applications to the investigation of boundary value problems for degenerate elliptic equations // Izv. Vyssh. Uchebn. Zaved. Mat. 8, 4-30 (1988). [Soviet Math. Izv. VUZ. Matem. 32:8, 1-40 (1988).]

2. N.V. Miroshin. Spectral exterior problems for a degenerate elliptic operator // Izv. VUZov. Matem. 8, 47-55 (1988). [Soviet Math. Izv. VUZ. 32:8, 64-74 (1988).]

3. N.V. Miroshin. The exterior Dirichlet variational problem for an elliptic operator with degeneration // Trudy Matem. Inst. im V.A. Steklova RAN. 194, 179-195 (1992). [Proc. Steklov Inst. Math. 194, 187-205 (1993).]

4. S.A. Iskhokov. On the smoothness of the solution of degenerate differential equations // Differ. Uravn. 31:4, 641-653 (1995). [Diff. Equat. 31:4, 594-606 (1995).]

5. S.A. Iskhokov, A.Ya. Kudzhmuratov. On the variational Dirichlet problem for degenerate elliptic operators // Doklady AN. 403:2, 165-168 (2005). [Doklady Math. 72:1, 512-515 (2005).]

6. S.A. Iskhokov. Gårding's inequality for elliptic operators with degeneracy // Matem. Zametki. 87:2, 201-216 (2010). [Math. Notes. 87:2, 189-203 (2010).]

7. S.A. Iskhokov, M.G. Gadoev, I.A. Yakushev. Gårding inequality for higher order elliptic operators with a non-power degeneration and its applications // Ufimskij Math. J. 8:1, 54-71. [Ufa Math. J. 8:1, 51-67 (2016).]

8. S.A. Iskhokov, O.A. Nematulloev. On solvability of variational Dirichlet problem with inhomogeneous boundary conditions for degenerate elliptic operators in bounded domain // Doklady AN Resp. Tajikistan. 56:5, 352-358 (2013). (in Russian).

9. K.Kh. Boimatov. The generalized Dirichlet problem for systems of second-order differential equations // Doklady AN. 327:1, 9-15 (1992). [Doklady Math. 46:3, 403-409 (1993).]

10. K.Kh. Bojmatov. The generalized Dirichlet problem connected with a noncoercive bilinear form // Doklady AN. 330:3, 285-290 (1993). [Russ. Acad. Sci. Doklady Math. 330:3, 455-463 (1993).]

11. K.Kh. Boimatov. Matrix differential operators generated by noncoercive bilinear forms // Doklady AN. 339:1, 5-10 (1994). [Doklady Math. 50:3, 351-359 (1995).]

12. K.Kh.Boimatov and S.A. Iskhokov. On solvability and smoothness of a solution of the variational Dirichlet problem associated with a noncoercive bilinear form // Trudy Mat. Inst. Steklova. 214, 101-127 (1996). [Proc. Steklov Inst. Math. 1996, 214, 101-127 (1996).]

13. S.A. Iskhokov. The variational Dirichlet problem for elliptic operators with nonpower degeneration generated by noncoercive bilinear forms // Doklady AN. 392:5, 606-609 (2003). [Doklady Math. 68:2, 261-265 (2003).]

14. S.A. Iskhokov, A.G. Karimov. On smoothness of solution of variational Dirichlet problem for elliptic operators associated with non-coercive bilinear forms // Doklady AN Resp. Tajikistan. 47:4, 68-74 (2004). (in Russian).

15. K.Kh. Boimatov. On the Abel basis property of the system of root vector-functions of degenerate elliptic differential operators with singular matrix coefficients // Sibir. Matem. Zhurn. 47:1, 46-57 (2006). [Siberian Math. J. 47:1, 35-44 (2006).]

16. S.A. Iskhokov, M.G. Gadoev, T. Konstantinova. Variational Dirichlet problem for degenerate elliptic operators generated by noncoercive forms // Doklady AN. 462:1, 7-10 (2015). [Doklady Math. 91:3, 255-258 (2015).]

17. M.G. Gadoev and T.P. Konstantinova. Solvability of the Dirichlet variational problem for a class of degenerate elliptic operators // Matem. Zamet. SBFU. 21:1, 8-21 (2014). [Yak. Math. J. 21:2, 6-18 (2014).]

18. S.A. Iskhokov, M.G. Gadoev, M.N. Petrova. On some spectral properties of a class of degenerate elliptic differential operators // Matem. Zamet. SVFU. 23:2, 31-50 (2016). (in Russian).

19. K.Kh. Boimatov. On the denseness of the compactly supported functions in weighted spaces // Doklady AN SSSR. 307:6, 1296-1299 (1989). [Soviet Math. Dokl. 40:6, 225-228 (1990).] 
20. S.A. Iskhokov. On the smoothness of a generalized solution of the Dirichlet variational problem for degenerate elliptic equations in a half-space // Doklady AN. 330:4, 420-423 (1993). [Doklady Math. 47:3, 504-508 (1993).]

21. L.D. Kudryavtsev. Imbedding theorems for functions defined on unbounded regions // Doklady AN SSSR. 153:3, 530-532 (1963). [Sov. Math. Dokl. 4, 1715-1717 (1963).]

22. T.S. Pigolkina. Density of finite functions in weight classes // Matem. Zamet. 2:1, 53-60 (1967). [Math. Notes. 2:1, 518-522 (1967).]

23. Yu.M. Berezansky. Expansion over eigenfunctions of self-adjoint operators. Naukova Dumka, Kiev (1965). (in Russian).

24. K.Kh. Boimatov. The spectral asymptotics of differential and pseudo-differential operators. I. // Tr. Semin. Im. I. G. Petrovskogo. 7, 50-100 (1981). (in Russian).

25. K.Kh. Boimatov. Separability theorems, weighted spaces and their applications // Trudy Matem. Inst. Stekl. AN SSSR. 170, 37-76 (1984). [Proc. Steklov Inst. Math. 170, $39-81$ (1987).]

26. M.Sh. Birman, M.Z. Solomyak. Spectral theory of self-adjoint operators in Hilbert space. Izd-vo Leningrad. Univ., Leninfrad (1980). [D. Reidel Publ. Comp., Dordrecht (1987).]

27. T. Kato. Perturbation theory of linear operators. Springer-Verlag, Berlin (1967).

28. S.A. Iskhokov. On the smoothness of solutions of the generalized Dirichlet problem and the eigenvalue problem for differential operators generated by noncoercive bilinear forms // Doklady AN. 342:1, 20-22 (1995). [Doklady Math. 51:3, 323-325 (1995).]

Sulaimon Abunasrovich Iskhokov,

Institute of Mathematics named after A. Dzhuraev AS RT,

Aini str. 299/4,

734063, Dushanbe, Tadjikistan,

Mirny Polytechnic Institute,

a branch of North-Eastern Federal University named after M.K. Ammosov,

Tikhonova str., 5/1,

678170, Mirny, Republic of Sakha (Yakutia), Russia

E-mail: sulaimon@mail.ru

Bakhtovar Abduganievich Rakhmonov,

Institute of Mathematics named after A. Dzhuraev AS RT,

Aini str. 299/4,

734063, Dushanbe, Tadjikistan

E-mail: bakhtovar-1989@mail.ru 